\title{
Correlation between the Incidence and Attributable Mortality Fraction of Acute Kidney Injury: A Systematic Review
}

\author{
Yohei Komaru $^{\text {a }}$ Ryota Inokuchi ${ }^{\text {b }}$ Masao Iwagami ${ }^{c}$ Ryo Matsuura ${ }^{a}$ \\ Yoshifumi Hamasaki $^{d}$ Masaomi Nangaku $^{\text {a,d }}$ Kent Doi $^{\text {b }}$ \\ a Division of Nephrology and Endocrinology, Graduate School of Medicine, The University of Tokyo, Tokyo, Japan; \\ ${ }^{b}$ Department of Acute Medicine, The University of Tokyo Hospital, Tokyo, Japan; ' Department of Health Services \\ Research, University of Tsukuba, Tsukuba, Japan; ${ }^{d}$ Division of Hemodialysis and Apheresis, The University of Tokyo \\ Hospital, Tokyo, Japan
}

\section{Keywords}

Acute kidney injury $\cdot$ Acute renal failure $\cdot$ Incidence .

Attributable fraction of mortality

\begin{abstract}
Introduction: The incidence of acute kidney injury (AKI) as diagnosed by international standardized criteria as well as its mortality has undergone extreme variations. Although AKI is a significant worsening mortality factor, a higher prevalence may lead to better patient management, thereby lowering mortality. We investigated the correlation between AKI incidence and its associated mortality. Methods: We conducted a systematic review of studies on AKI reporting its incidence and mortality. Literature searches were performed in MEDLINE, EMBASE, and Cochrane Library, within the time frame of 2004-2018. Studies with small number of participants ( $<500$ for adult cohorts, 50 for pediatric cohorts) were excluded. The correlation among AKI incidence, mortality, and AKI-attributable fraction of mortality was evaluated using a regression model. The trend test was used to analyze the effect of publication year and country gross domestic product (GDP). Results: A total of 4,694 manuscripts were screened, from which 287 cohorts were eligible (adults: 203
\end{abstract}

cohorts comprising 7,076,459 patients; children: 84 comprising 69,677 patients). Within adult cohorts, AKI patients' mortality increased $\left(R^{2}=0.023, \beta=0.12, p=0.03\right)$ but the attributable fraction of mortality decreased $\left(R^{2}=0.27, \beta=-0.43\right.$, $p<0.001)$ with the increasing AKI incidence. Both more recent publications and higher GDP countries had a lower crude AKI patients' mortality, although AKI-attributable fraction did not decrease. Conclusions: Cohorts with high AKI incidence had a relatively low AKI-attributable mortality fraction, which suggests an advantage of more experienced AKI management. Further study is needed, however, to address the heterogeneity of included cohorts and to confirm the causality. (Registered in prospective register of systematic reviews database; CRD 42019129322.$)$

(c) 2020 S. Karger AG, Basel

\section{Introduction}

Acute kidney injury (AKI) has been increasingly recognized in clinical practice as a high burden disease. Annually, about 13.3 million people worldwide are thought to suffer from AKI, with 10 to $15 \%$ of them dying $[1,2]$. Although considerable efforts have been made to tackle 
this global issue, the challenge to eradicate preventable AKI-attributable deaths remains halfway [3].

AKI diagnosis and grading based on international standardized criteria - the Risk-Injury-Failure-Loss of kidney function-end stage kidney disease (RIFLE) [4], AKI network (AKIN) [5], and kidney disease: improving global outcomes (KDIGO) [6] criteria-enabled us to compare the AKI incidence and prognosis in different clinical settings. Both AKI incidence and prognosis varied significantly. A systematic review and meta-analysis on AKI-related reports published during 2004-2012 estimated the pooled AKI incidence and mortality rates worldwide as 21.6 and $23.9 \%$, respectively [7]. This review also looked into the association of AKI mortality with the publication year and the country gross domestic product (GDP), revealing that mortality declined with later publication year and higher GDP.

Although the number of reports on AKI increased rapidly after the preceding review, investigations on the high variability of AKI incidence and mortality remain insufficient. Specifically, no study has addressed the AKI-attributable fraction of mortality. AKI patients' prognosis depends not only on the disease's etiology but also on the methods of diagnosis and management used in each medical setting [8]. Recent studies further proved that a larger hospital volume or case volume leads to better clinical outcomes in patients with various diseases, such as sepsis [9, $10]$, leukemia [11], and those requiring surgery $[12,13]$.

The present study aimed to report the correlation among AKI incidence, mortality, and AKI-attributable fraction of mortality. We hypothesized that a higher AKI incidence, reflecting a higher case volume and experience per physician, would be associated with lower mortality.

\section{Methods}

\section{Study Design and Search Strategy}

The present study is a systematic review of published manuscripts addressing AKI's incidence and mortality in certain cohorts. The study protocol was registered in the international prospective register of systematic reviews database (registration number, CRD 42019129322). Eligible studies were those that adopted any KDIGO-equivalent AKI criteria (either RIFLE, AKIN, or KDIGO). Basic search terms were identical to those used in the previous systematic review (online suppl. Table S1; for all online suppl. material, see www.karger.com/doi/10.1159/000505568) [7]. Briefly, the search strategy comprised a combination of terms indicative of the clinical AKI status (such as "AKI," "acute renal failure," and "acute tubular necrosis") and its diagnostic criteria (such as "risk," "injury," "failure," "rifle," "akin," and "kdigo"). Exclusion criteria were as follows: (1) lack of KDIGO-equivalent AKI definition, (2) absence of comparable non-AKI cohort, (3) no mortality data of AKI and/or non-AKI group, (4) lack of short term (30 days and less) outcome evaluation, (5) small sample size (for adult studies: <500; for pediatric studies: <50), (6) case-control studies or case reports, (7) second reports on the same cohort, and (8) studies not written in English language.

\section{Information Sources}

Data screening within the time frame of 2004 to August 23, 2012, was performed referring to the supplementary reference list of the previous systematic review [7]. Studies published between August 24, 2012, and May 1, 2018, were then searched in MEDLINE, EMBASE, and Cochrane Library on May 15, 2018.

\section{Quality Assessment of each Study}

All studies that satisfied the inclusion criteria were evaluated for its quality, using the Newcastle-Ottawa quality scale for cohort studies [14]. Study selection and quality assessment were performed by 2 independent physicians (Y.K. and R.I.; Y.K. and M.I.), and any discrepancies between them were discussed with a third physician (K.D.) to reach the final decision.

\section{Data Extraction}

Data from each eligible study were extracted by pair of 2 individual reviewers; both independently used standardized spread sheet for this process. Data extracted from the full text review included each study's characteristics (study design, publication year, clinical settings, used AKI criteria, country of origin), participants' demographics (sample size, mean age, proportion of women, the number of patients with and without AKI, and the number of patients by AKI severity). Primary outcomes included $<30$-day mortality, in-hospital mortality, or in-intensive care unit mortality. We extracted mortality of those with and without AKI in each cohort and mortality by AKI severity, if available. AKI etiologies were divided into subgroups as follows: critical care; cardiovascular surgery; major surgery; post-acute coronary syndrome; infection including sepsis; liver disease; trauma; heart failure; nephrotoxins; hematology, oncology; mixed and others. The discrepancies between 2 reviewers were consulted to third reviewer to reach consensus.

\section{Data Synthesis}

The AKI-attributable fraction of mortality was calculated based on the mortality of patients with and without AKI in each study cohort, using following formula $[15,16]$ :

AKI - attributable fraction of mortality

= (mortality of AKI patients) - (mortality of non AKI patients)/ (mortality of AKI patients)

$=1-(\text { relative risk of death for } A K I)^{-1}$

This index ranged from 0 to 1 and expressed the proportion of AKI patients whose death was attributable to AKI.

We conducted a subgroup analysis for different AKI severities. AKI cohorts with severity data using KDIGO-equivalent AKI criteria were divided into 2 subgroups of mild and severe AKI; mild AKI corresponded to "Risk" category in RIFLE criteria and stage 1 in AKIN and KDIGO criteria, while severe AKI corresponded to "Injury" and "Failure" category in RIFLE criteria, and stages 2 and 3 in AKIN and KDIGO criteria.

The international monetary fund electronic database was consulted to acquire each country's latest GDP per capita on May, 2018 [17]. Whenever a cohort had 2 countries of origin, the aver- 
Fig. 1. Search process and literature selection. AKI, acute kidney injury; KDIGO, Kidney Disease Improving Global Outcomes.

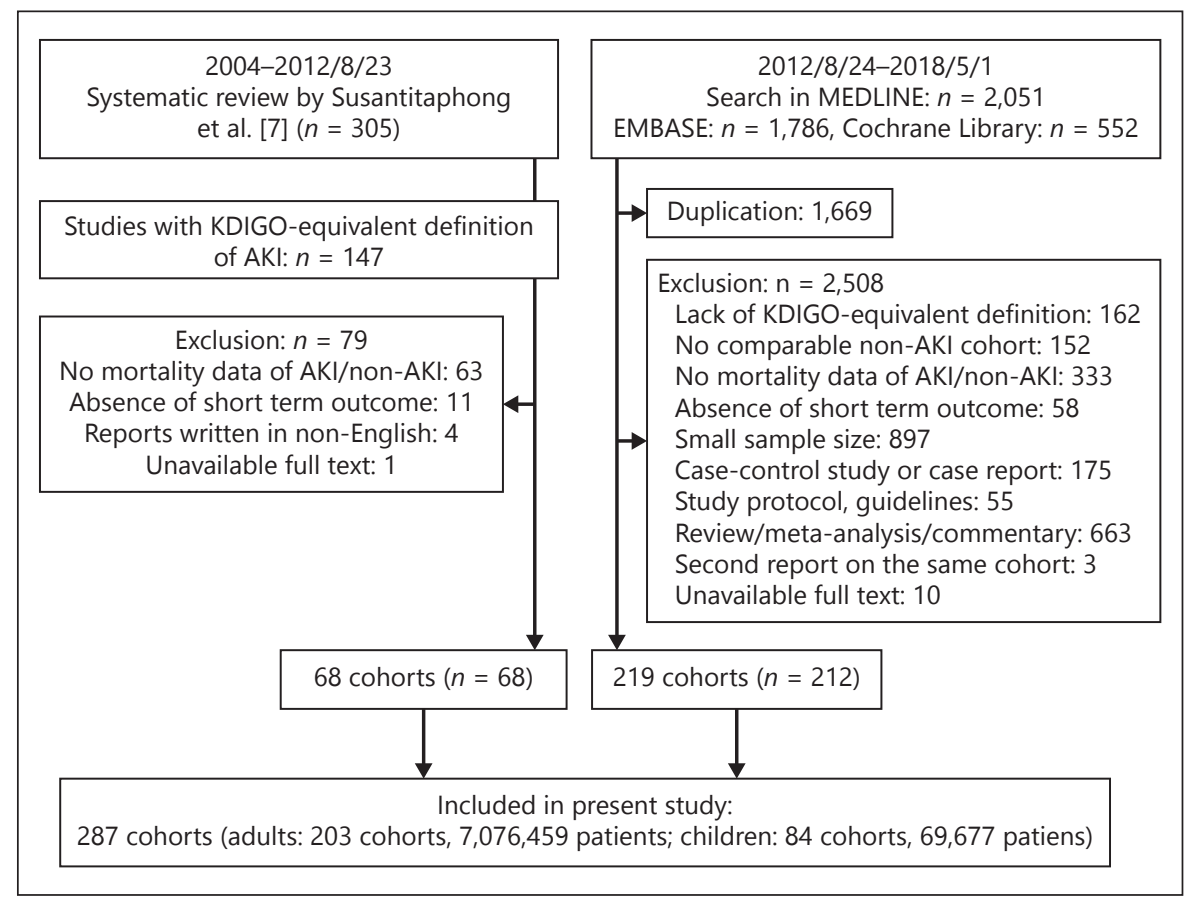

age GDP of the 2 countries was adopted for that cohort. Whenever a cohort had $>2$ countries of origin, the study was excluded from any analyses using GDP. Aggregated cohorts were divided into 4 groups according to the GDP's quartiles.

\section{Statistical Analyses}

First, the characteristics of the included cohorts were aggregated. Continuous parameters were expressed as mean $\pm \mathrm{SD}$, whereas categorical variables were expressed as frequencies and percentages. Second, the association between AKI patients' incidence and mortality within each cohort was investigated. The regression coefficient $(\beta)$ and the squared correlation coefficient $\left(R^{2}\right)$ were calculated using the least-square approach. Subsequently, the least-square approach weighted with each cohort's population size was performed. The association between AKI incidence and AKIattributable fraction of mortality was similarly investigated. Finally, the impact of publication year and GDP on AKI mortality and AKI-attributable fraction of mortality was examined in adult cohorts. The Jonckheere-Terpstra's trend test was performed to validate the associations' trend. All $p$ values reported in this manuscript were two-sided, and a level of $p<0.05$ was considered statistically significant. The software JMP pro version 13 (SAS institute Inc., Cary, NC, USA) and SPSS version 23 (IBM Corp., Armonk, NY, USA) were used for the statistical analyses.

\section{Results}

Systematic Review and Characteristics of the Included Cohorts

Among the 305 studies included in the previous systematic review [7], 68 met our inclusion criteria (Fig. 1).
The subsequent systematic literature searches performed in MEDLINE, EMBASE, and Cochrane Library yielded $2,051,1,786$, and 552 potentially relevant reports, respectively. A total of 4,177 reports were excluded for meeting at least one of the exclusion criteria (Fig. 1). Two studies retrieved by MEDLINE contained $>1$ independent eligible cohorts. As a result, a total of 287 cohorts from 280 studies published during 2007-2018 were included in the present study; 203 were adult cohorts comprising $7,076,459$ patients, and 84 were pediatric cohorts comprising 69,677 patients. A full reference list of the included manuscripts is available in the supplementary material (online suppl. Table S2). The quality of included cohort studies varied from point of 5 to 9 in the Newcastle-Ottawa scale for cohorts studies; out of the 280 studies, none was classified as poor quality (point 0-3), $142(50.7 \%)$ were fair quality (4-6), and 138 (49.3\%) were good quality (7-9).

The characteristics of the included cohorts are summarized in Table 1 . The average age of adults and pediatric cohorts were 64.4 and 4.3 years, respectively. Around $70 \%$ of participants in the adult cohorts and $55 \%$ in the pediatric cohorts were male. The average AKI proportion was 29.1 and $37.8 \%$ for adults and children, respectively. The countries of origin were distributed worldwide, with studies from Europe, North America, and Asia accounting for about $30 \%$ each. More eligible cohorts were found among more recent publications than among those pub- 
Table 1. Characteristics of the included cohorts

\begin{tabular}{|c|c|c|}
\hline & Adult cohorts $(n=203)$ & Paediatric cohorts $(n=84)$ \\
\hline Total population & $7,076,459$ & 69,677 \\
\hline Number of cohorts & 203 & 84 \\
\hline Age, years, mean \pm SD & $64.4 \pm 4.5$ & $4.3 \pm 2.6$ \\
\hline Gender, male, $\%$, mean \pm SD & $68.9 \pm 15.0$ & $55.2 \pm 3.6$ \\
\hline AKI proportion (per cohort), $\%$, mean \pm SD & $29.1 \pm 18.5$ & $37.8 \pm 19.9$ \\
\hline \multicolumn{3}{|l|}{ Publication year, $n(\%)$} \\
\hline 2007-2009 & $21(10.3)$ & $5(6.0)$ \\
\hline $2010-2012$ & $40(19.7)$ & $9(10.7)$ \\
\hline $2013-2015$ & $87(42.9)$ & $33(39.3)$ \\
\hline $2016-2018$ & $55(27.1)$ & $37(44.0)$ \\
\hline \multicolumn{3}{|l|}{ Region (cohorts), $n(\%)$} \\
\hline Europe & $63(31.0)$ & $11(13.1)$ \\
\hline North America & $57(28.1)$ & $36(42.9)$ \\
\hline Asia & $55(27.1)$ & $24(28.6)$ \\
\hline Oceania & $8(3.9)$ & $0(0)$ \\
\hline South America & $6(3.0)$ & $9(10.7)$ \\
\hline Africa & $1(0.5)$ & $1(1.2)$ \\
\hline Multicontinents & $13(6.4)$ & $3(3.6)$ \\
\hline \multicolumn{3}{|l|}{ Study design, $n(\%)$} \\
\hline Retrospective cohort & $158(77.8)$ & $55(65.5)$ \\
\hline Prospective cohort & $45(22.2)$ & $29(34.5)$ \\
\hline \multicolumn{3}{|l|}{ AKI diagnostic criteria, $n(\%)$} \\
\hline RIFLE & $64(31.5)$ & $41(48.8)$ \\
\hline AKIN & $67(33.0)$ & $10(11.9)$ \\
\hline KDIGO & $72(35.5)$ & $33(39.3)$ \\
\hline \multicolumn{3}{|l|}{ AKI settings, $n(\%)$} \\
\hline Critical care & $66(32.5)$ & $44(52.4)$ \\
\hline Cardiovascular surgery & $44(21.7)$ & $22(26.2)$ \\
\hline Major surgery & $24(11.8)$ & $0(0)$ \\
\hline Post AMI, PCI & $12(5.9)$ & $0(0)$ \\
\hline Infection, sepsis & $8(3.9)$ & $3(3.6)$ \\
\hline Liver disease & $6(3.0)$ & $2(2.4)$ \\
\hline Trauma & $4(2.0)$ & $1(1.2)$ \\
\hline Heart failure & $4(2.0)$ & $3(3.6)$ \\
\hline Nephrotoxins & $3(1.5)$ & $5(6.0)$ \\
\hline Hematology, oncology & $1(0.5)$ & $0(0)$ \\
\hline Mixed, others & $31(15.3)$ & $4(4.8)$ \\
\hline GDP/capita ( $\times$ USD $1,000 /$ year $)$, mean \pm SD & $43.3 \pm 20.7$ & $36.9 \pm 24.1$ \\
\hline
\end{tabular}

AKI, acute kidney injury; RIFLE, Risk-Injury-Failure-Loss of kidney function-end-stage kidney disease; AKIN, AKI network; KDIGO, Kidney Disease Improving Global Outcomes; AMI, acute myocardial infarction; PCI, percutaneous coronary intervention; GDP, gross domestic product.

lished a decade ago. The study design, used AKI criteria, and clinical settings of AKI are also described in Table 1. Based on the number of cohorts, critical care- and cardiovascular surgery-associated AKI were 2 major etiologies.

\section{AKI Incidence, AKI Mortality, and AKI-Attributable Fraction of Mortality}

The association between each cohort's AKI incidence and mortality is presented in Figure 2a, b. Within adult cohorts, mortality increased as the AKI incidence became higher, although the correlation coefficient was low $\left(R^{2}=\right.$ $0.023, \beta=0.12, p=0.033$, Fig. 2a). Subsequent analysis weighted with the number of patients in each adult cohort $\left(R^{2}=0.22, \beta=0.36, p<0.001\right)$ further confirmed this significant association. No such associations were observed in pediatric cohorts (Fig. 2b).

Next, the association between AKI incidence and AKIattributable fraction of mortality was investigated. As 


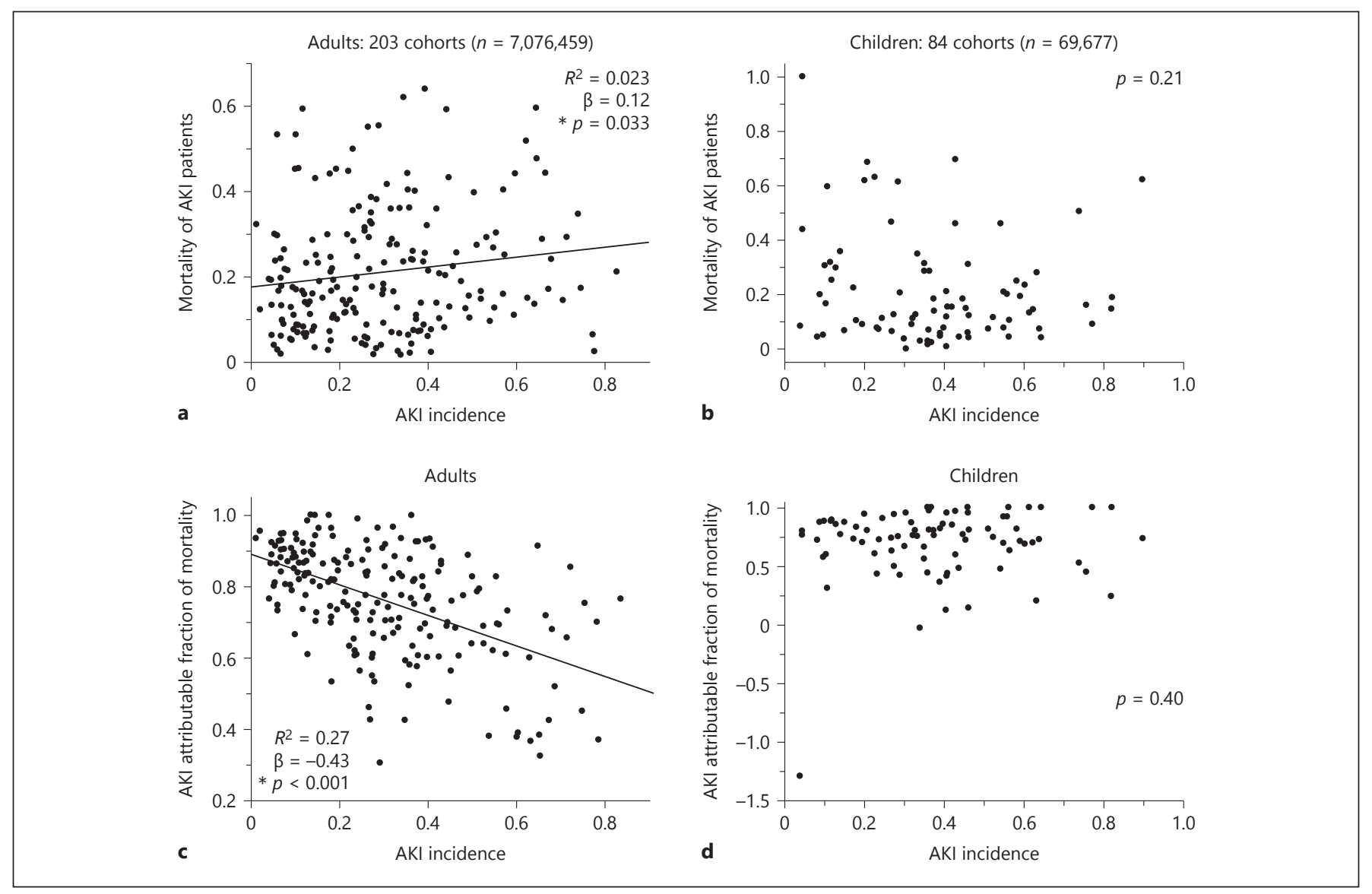

Fig. 2. Association between AKI incidence and mortality within each cohort. The incidence and crude mortality of AKI patients in adults (a) and children (b). Higher mortality was observed in adult cohorts with a higher AKI incidence $(p=0.03$, a). The incidence and AKI-attributable fraction of mortality is shown in adult (c)

shown in Figure $2 \mathrm{c}$, d, the AKI-attributable fraction of mortality decreased significantly as the AKI incidence became higher in the adult cohorts $\left(R^{2}=0.27, \beta=-0.43, p<\right.$ 0.001 , Fig. $2 c)$. The association was also reproduced in subsequent analysis weighted with sample size $\left(R^{2}=0.49\right.$, $\beta=-0.64, p<0.001)$. Again, these associations were not observed in children (Fig. 2d).

\section{Subgroup Analysis by AKI Severity on AKI Incidence and Mortality}

In the 287 included cohorts, 149 (51.9\%) had information of AKI severity and were included in subsequent subgroup analysis. As shown in online supplementary Figure 1 , there was no correlation between incidence and mortality of AKI both in mild and severe AKI among adult cohorts. However, the inverse correlation between AKI incidence and AKI-attributable fraction of mortality was still signifi- and in pediatric (d) cohorts. There was a decline of the attributable mortality in adult cohorts with higher AKI incidence $(p<0.001$, c). These associations were not observed in pediatric cohorts (b, d); ${ }^{*} p<0.05$. AKI, acute kidney injury.

cant even after the total AKI population was divided into mild and severe AKI $\left(R^{2}=0.25, \beta=-0.60, p<0.001\right.$, for mild AKI; $R^{2}=0.19, \beta=-0.35, p<0.001$, for severe AKI). These inverse correlations between AKI incidence and AKI-attributable fraction of mortality in subgroup analysis were also observed in pediatric cohorts $\left(R^{2}=0.13, \beta=-0.80, p=\right.$ 0.031 , for mild AKI; $R^{2}=0.09, \beta=-0.24, p=0.049$, for severe AKI) as shown in online supplementary Figure 2.

\section{Publication Year and GDP}

Within the adult cohorts, the mortality of AKI patients decreased significantly in more recent publications (Fig. 3a, $p=0.001$ ) and in reports from countries with higher GDP (Fig. 3b, $p=0.032$ ). However, no significant differences were observed in the AKI-attributable fraction of mortality neither over time (Fig. 3c) nor in the quantile GDP classes (Fig. 3d). 


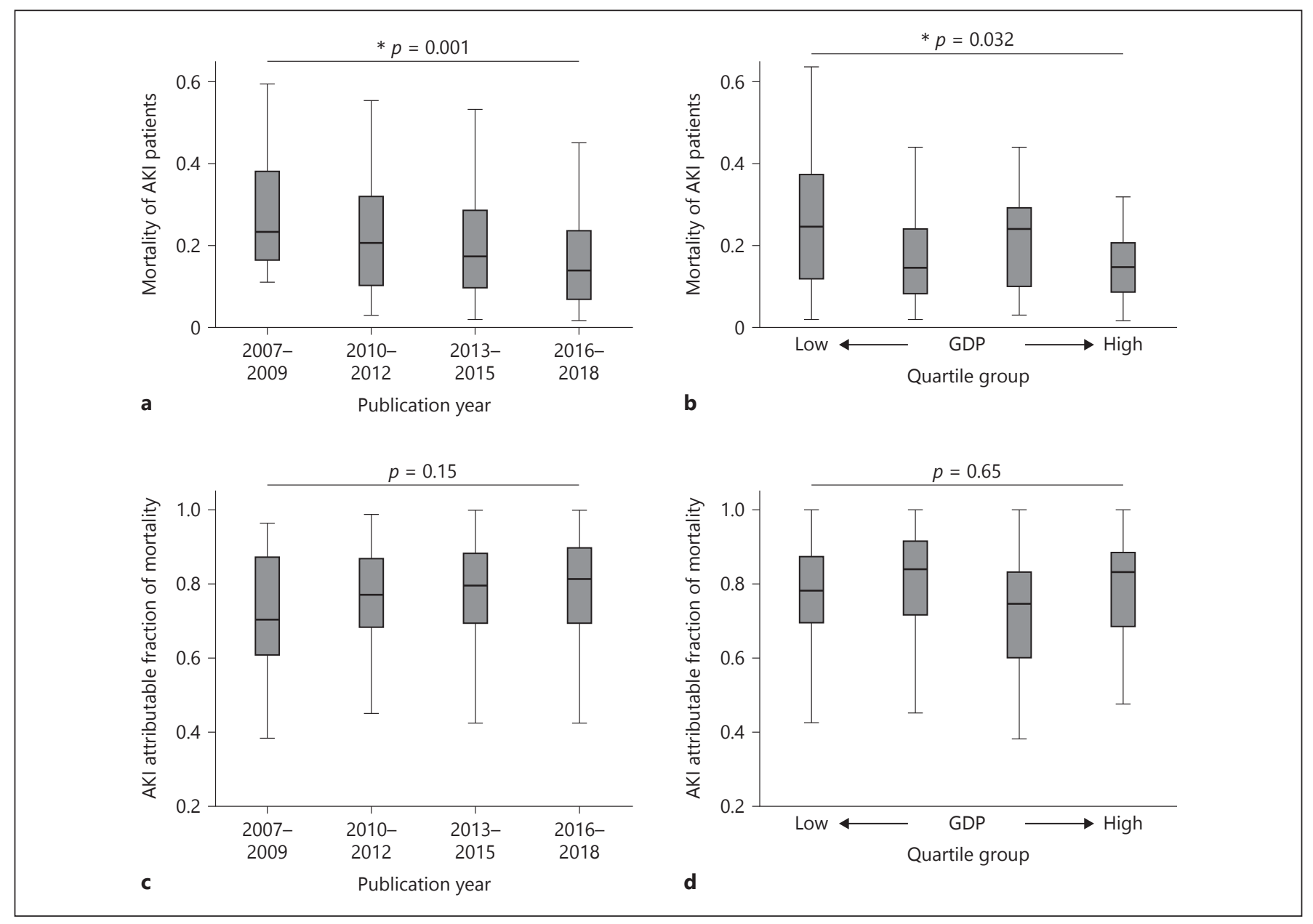

Fig. 3. The impact of publication year and national GDP on AKI mortality and AKI-attributable fraction of mortality. The mortality of AKI patients declined in more recent publications (a) and in countries with higher GDP (b). The AKI-attributable fraction of

\section{Discussion/Conclusion}

In the present study, the possible association between AKI incidence and mortality was investigated through a systematic review of previous reports from 2007 to 2018, comprising a total of 287 AKI cohorts. Although AKI patients' mortality was higher within cohorts with higher AKI incidence, the AKI-attributable fraction of mortality declined as the incidence of AKI increased, especially in adults. This decline of AKI-attributable fraction of mortality was also observed in subgroup analysis of both mild and severe AKI. Both a later publication year and a higher national GDP were associated with a lower crude mortality among AKI patients, although the AKI-attributable fraction did not show the same tendency.

mortality did not show significant differences as publication year (c) and GDP (d) changed. $p$ values were calculated by JonckheereTerpstra's trend test; * $p<0.05$. AKI, acute kidney injury; GDP, gross domestic product.

Overall, a higher AKI incidence suggests a more severe clinical condition of patients, leading to higher mortality. This is because kidney dysfunction frequently appears as a part of multiorgan dysfunction [18]. Our results are consistent with those of previous reports that showed that higher AKI incidence is accompanied by higher mortality $[19,20]$. On the contrary, the AKI-attributable fraction of mortality was inversely correlated with each cohort's AKI incidence (Fig. 2c). To our knowledge, this is the first systematic review introducing the index of AKIattributable fraction of mortality to estimate the impact of AKI on patient's outcome. In general, low AKI-attributable fraction of mortality means relatively low mortality of patients with AKI compared with those without AKI in each cohort. The decline of AKI-attributable frac- 
tion of mortality among cohorts with higher AKI incidence may reflect a better patient monitoring and more appropriate clinical management. For example, early detection of AKI by electronic alert systems leads to an increased incidence of AKI, without causing higher mortality [21-23]. In the present study, it was also notable that even patients with severe AKI showed lower AKI-attributable fraction of mortality in cohorts with higher AKI incidence, than those in cohorts with lower AKI incidence (online suppl. Fig. 1d). It may propose that local facilities with higher AKI incidence provide better management with more experienced medical professionals and enough medical resources [24]. Conversely, our results suggest that patients in low AKI incidence cohorts require closer monitoring, as the AKI-attributable fraction of mortality in such cohorts was considerably high.

Further investigation on the generalizability of the inverse association between AKI incidence and the AKI-attributable fraction of mortality is needed. Since a previous report denied the effect of hospital case volume on patients with AKI treated with dialysis [25], the favorable effect of higher AKI incidence determined by our study may occur specifically among nondialysis AKI patients. Moreover, it was noted that similar analysis of total pediatric AKI cohorts did not show significant trends, probably due to a limited number of reports and different clinical settings.

A previous systematic review [7] showed that a lower mortality among AKI patients was associated with later publication years and higher national GDP. This trend was reproduced by our study including more recent publications (Fig. 3a, b). However, neither later publication years nor higher GDP lowered the AKI-attributable fraction of mortality (Fig. 3c, d). These results suggest that the overall survival of patients with and without AKI improved during the observation period. However, effective and specific AKI treatment might not have been available even in recent years and in high-income countries. Future developments in AKI management, such as care bundle introduction [26,27] and novel pharmaceutical approaches, may lower the AKI-attributable fraction of mortality.

We acknowledge several limitations in the present study. First, most included studies were observational, among which more than half were retrospective cohort studies. The cohorts contained considerable heterogeneity and various confounders, and the outcome varied among studies. Given these settings, we aggregated a large number of studies and participants, although residual confounds were inevitable. Second, studies with a small number of participants $(<500$ for adult cohorts and 50 for pediatric cohorts) were excluded, leading to the exclusion of most randomized trials and studies from developing countries. Moreover, the possibility of publication bias was not sufficiently ruled out; AKI cohorts with low AKI incidence and low mortality may not be reported given their low clinical impact. Future exhaustive surveys are therefore needed to address these issues.

Since the standard AKI criteria were developed, the AKI incidence and mortality reported in global studies underwent an extreme variation. Although patients' mortality was higher in the cohorts with higher AKI incidence, the AKI-attributable fraction of mortality, which indicates the clinical impact of AKI on death, decreased as the incidence increase. These results may indicate the advantage of a more experienced AKI management; however, it should be noted most enrolled studies were retrospective observational and causality cannot be confirmed.

\section{Acknowledgments}

The authors would like to thank Enago (www.enago.jp) for the English language review.

\section{Statement of Ethics}

We conducted this study in accordance with the tenets of the World Medical Association Declaration of Helsinki. The requirement for written informed consent was not applicable because of the nature of systematic review.

\section{Disclosure Statement}

The authors have no conflicts of interest to declare.

\section{Funding Sources}

The present research did not receive any financial support from external organization.

\section{Author Contributions}

Y.K. and K.D. designed this study. Y.K., R.I., and M.I. conducted literature search, data extraction, and subsequent analyses. Y.K., R.M., and K.D. validated and interpreted the results. Y.K. drafted the manuscript, R.I., K.D., Y.H., and M.N. reviewed and edited. All the authors read through the manuscript and agreed to the submission of the final version. 


\section{References}

1 Lewington AJ, Cerdá J, Mehta RL. Raising awareness of acute kidney injury: a global perspective of a silent killer. Kidney Int. 2013 Sep; 84(3):457-67.

2 Chawla LS, Kimmel PL. Acute kidney injury and chronic kidney disease: an integrated clinical syndrome. Kidney Int. 2012 Sep; 82(5):516-24.

3 Mehta RL, Cerdá J, Burdmann EA, Tonelli M, García-García G, Jha V, et al. International Society of Nephrology's 0by25 initiative for acute kidney injury (zero preventable deaths by 2025): a human rights case for nephrology. Lancet. 2015 Jun;385(9987):2616-43.

4 Bellomo R, Ronco C, Kellum JA, Mehta RL, Palevsky P; Acute Dialysis Quality Initiative workgroup. Acute renal failure - definition, outcome measures, animal models, fluid therapy and information technology needs: the Second International Consensus Conference of the Acute Dialysis Quality Initiative (ADQI) Group. Crit Care. 2004 Aug;8(4): R204-12.

5 Mehta RL, Kellum JA, Shah SV, Molitoris BA, Ronco C, Warnock DG, et al.; Acute Kidney Injury Network. Acute Kidney Injury Network: report of an initiative to improve outcomes in acute kidney injury. Crit Care. 2007; 11(2):R31.

6 Kidney Disease: Improving Global Outcomes (KDIGO): Clinical Practice Guideline for Acute Kidney Injury. Kidney Int Suppl. 2012; 2:1-138.

7 Susantitaphong P, Cruz DN, Cerda J, Abulfaraj M, Alqahtani F, Koulouridis I, et al.; Acute Kidney Injury Advisory Group of the American Society of Nephrology. World incidence of AKI: a meta-analysis. Clin J Am Soc Nephrol. 2013 Sep;8(9):1482-93.

8 Hoste EA, Kellum JA, Selby NM, Zarbock A, Palevsky PM, Bagshaw SM, et al. Global epidemiology and outcomes of acute kidney injury. Nat Rev Nephrol. 2018 Oct;14(10):60725.

9 Gaieski DF, Edwards JM, Kallan MJ, Mikkelsen ME, Goyal M, Carr BG. The relationship between hospital volume and mortality in severe sepsis. Am J Respir Crit Care Med. 2014 Sep;190(6):665-74.
10 Gu WJ, Wu XD, Zhou Q, Zhang J, Wang F, Ma ZL, et al. Relationship between Annualized Case Volume and Mortality in Sepsis: A Dose-Response Meta-analysis. Anesthesiology. 2016 Jul;125(1):168-79.

11 Thompson MP, Waters TM, Kaplan EK, McKillop CN, Martin MG. Hospital volume and acute myeloid leukemia mortality in Medicare beneficiaries aged 65 years and older. Blood. 2016 Aug;128(6):872-4.

12 Tchouta LN, Park HS, Boffa DJ, Blasberg JD, Detterbeck FC, Kim AW. Hospital Volume and Outcomes of Robot-Assisted Lobectomies. Chest. 2017 Feb;151(2):329-39.

13 Zevin B, Aggarwal R, Grantcharov TP. Volume-outcome association in bariatric surgery: a systematic review. Ann Surg. 2012 Jul; 256(1):60-71

14 Wells GA, Shea B, O'Connell D, Peterson J, Welch V, Losos M, et al. The Newcastle-Ottawa Scale (NOS) for assessing the quality if nonrandomized studies in meta-analyses. [last accessed Oct 30, 2019]. Available from: http://www.ohri.ca/programs/clinical_ epidemiology/oxford.htm.

15 Flegal KM, Graubard BI, Williamson DF, Gail $\mathrm{MH}$. Impact of smoking and preexisting illness on estimates of the fractions of deaths associated with underweight, overweight, and obesity in the US population. Am J Epidemiol. 2007 Oct;166(8):975-82.

16 Shankar-Hari M, Harrison DA, Rowan KM, Rubenfeld GD. Estimating attributable fraction of mortality from sepsis to inform clinical trials. J Crit Care. 2018 Jun;45:33-9.

17 International Monetary Fund. IMF DataMapper, 2018 [cited May 15, 2018]. Available from: https://www.imf.org/external/ datamapper/datasets.

18 Peters E, Antonelli M, Wittebole X, Nanchal R, François B, Sakr Y, et al. A worldwide multicentre evaluation of the influence of deterioration or improvement of acute kidney injury on clinical outcome in critically ill patients with and without sepsis at ICU admission: results from The Intensive Care Over Nations audit. Crit Care. 2018 Aug;22(1):188.

19 Nisula S, Kaukonen KM, Vaara ST, Korhonen AM, Poukkanen M, Karlsson S, et al.; FINNAKI
Study Group. Incidence, risk factors and 90day mortality of patients with acute kidney injury in Finnish intensive care units: the FINNAKI study. Intensive Care Med. 2013 Mar;39(3):420-8.

20 Fujii T, Uchino S, Doi K, Sato T, Kawamura T; JAKID study group. Diagnosis, management, and prognosis of patients with acute kidney injury in Japanese intensive care units: the JAKID study. J Crit Care. 2018 Oct;47: 185-91.

21 Wilson FP, Shashaty M, Testani J, Aqeel I, Borovskiy Y, Ellenberg SS, et al. Automated, electronic alerts for acute kidney injury: a single-blind, parallel-group, randomised controlled trial. Lancet. 2015 May;385(9981): 1966-74.

22 Park S, Baek SH, Ahn S, Lee KH, Hwang H, Ryu J, et al. Impact of Electronic Acute Kidney Injury (AKI) Alerts With Automated Nephrologist Consultation on Detection and Severity of AKI: A Quality Improvement Study. Am J Kidney Dis. 2018 Jan;71(1):9-19.

23 Al-Jaghbeer M, Dealmeida D, Bilderback A, Ambrosino R, Kellum JA. Clinical Decision Support for In-Hospital AKI. J Am Soc Nephrol. 2018 Feb;29(2):654-60.

24 Ronco C, Rizo-Topete L, Serrano-Soto M, Kashani K. Pro: Prevention of acute kidney injury: time for teamwork and new biomarkers. Nephrol Dial Transplant. 2017 Mar;32(3): 408-13.

25 Chimunda T, Silver SA, Kuwornu JP, Li L, Nash DM, Dixon SN, et al. Hospital case volume and clinical outcomes in critically ill patients with acute kidney injury treated with dialysis. J Crit Care. 2018 Dec;48:276-82.

26 Meersch M, Schmidt C, Hoffmeier A, Van Aken H, Wempe C, Gerss J, et al. Prevention of cardiac surgery-associated AKI by implementing the KDIGO guidelines in high risk patients identified by biomarkers: the PrevA$\mathrm{KI}$ randomized controlled trial. Intensive Care Med. 2017 Nov;43(11):1551-61.

27 Göcze I, Jauch D, Götz M, Kennedy P, Jung B, Zeman F, et al. Biomarker-guided Intervention to Prevent Acute Kidney Injury After Major Surgery: The Prospective Randomized BigpAK Study. Ann Surg. 2018 Jun;267(6):1013-20. 